\title{
HAUSDORFF DIMENSION OF SETS WITH RESTRICTED, SLOWLY GROWING PARTIAL QUOTIENTS
}

\author{
HIROKI TAKAHASI
}

\begin{abstract}
I. J. Good (1941) showed that the set of irrational numbers in $(0,1)$ whose partial quotients $a_{n}$ tend to infinity is of Hausdorff dimension $1 / 2$. A number of related results impose restrictions of the type $a_{n} \in B$ or $a_{n} \geq f(n)$, where $B$ is an infinite subset of $\mathbb{N}$ and $f$ is a rapidly growing function with $n$. We show that, for an arbitrary $B$ and an arbitrary $f$ with values in $[\min B, \infty)$ and tending to infinity, the set of irrational numbers in $(0,1)$ such that

$$
a_{n} \in B, a_{n} \leq f(n) \text { for all } n \in \mathbb{N} \text {, and } a_{n} \rightarrow \infty \text { as } n \rightarrow \infty
$$

is of Hausdorff dimension $\tau(B) / 2$, where $\tau(B)$ is the exponent of convergence of $B$.
\end{abstract}

\section{InTRODUCTION}

Let $\mathbb{I}$ denote the set of irrational numbers in $(0,1)$. Each $x \in \mathbb{I}$ has an infinite continued fraction expansion $x=\left[a_{1}(x), a_{2}(x), \ldots\right]=1 /\left(a_{1}(x)+1 /\left(a_{2}(x)+\cdots\right)\right)$, where the positive integer $a_{n}(x)$ is called a partial quotient of $x$. Sets of irrational numbers whose partial quotients obey various conditions have been studied since the works of Jarník [11] and Good [7]. Among a number of results Good obtained in [7, the principal one states that

$$
\operatorname{dim}_{H}\left\{x \in \mathbb{I}: \lim _{n \rightarrow \infty} a_{n}(x)=\infty\right\}=\frac{1}{2},
$$

where $\operatorname{dim}_{H}$ denotes the Hausdorff dimension, see [7, Theorem 1]. Some refinements and extensions of Good's result are available. Ramharter [17] showed that the set of numbers in $\mathbb{I}$ with strictly monotone increasing partial quotients is of Hausdorff dimension 1/2. Jaerisch and Kesseböhmer [10] considered the set of numbers in $\mathbb{I}$ whose all partial quotients are greater than $q$, and obtained a precise asymptotics of the Hausdorff dimension of this set as $q \rightarrow \infty$ using the thermodynamic formalism. Jordan and Rams [12] extended Ramharter's result to some iterated function systems.

In [9], Hirst proved results analogous to that of Good concerning the cases where $a_{n}$ is restricted to belong to some sequence of natural numbers. For an infinite subset $B$ of $\mathbb{N}=\{1,2, \ldots\}$, define

$$
E(B)=\left\{x \in \mathbb{I}: a_{n}(x) \in B \text { for all } n \in \mathbb{N}, \text { and } \lim _{n \rightarrow \infty} a_{n}(x)=\infty\right\},
$$

2010 Mathematics Subject Classification. 11A55, 11K50, 37A45.

Keywords: continued fractions, partial quotients, Hausdorff dimension. 
and define the exponent of convergence of $B$ by

$$
\tau(B)=\inf \left\{s \geq 0: \sum_{k \in B} k^{-s}<\infty\right\} .
$$

Hirst showed that $\operatorname{dim}_{H} E(B) \leq \tau(B) / 2$ (see [9, Corollary 1]), and conjectured that the equality holds for an arbitrary $B$. He treated the special case $B=\left\{k^{b}\right\}_{k \in \mathbb{N}}$, $b$ a positive integer to support his conjecture (see [9, Theorem 3]). Cusick [3, Theorem 1] proved that the equality holds in the case $B$ is not too sparse and satisfies what he called the density assumption. After 35 years of the appearance of Hirst's paper [9], Wang and Wu solved the conjecture of Hirst in the affirmative, see [20, Theorem 1.1]. For an extension of Wang and Wu's result to iterated function systems, see [1].

The Hausdorff dimension of sets of the form

$$
F(B, f)=\left\{x \in E(B): a_{n}(x) \geq f(n) \text { for all } n \in \mathbb{N}\right\},
$$

where $f$ is any function which tends to infinity with $n$ has also been considered in the literature. In [9, p.227], Hirst conjectured that the equality $\operatorname{dim}_{H} F(B, f)=$ $\tau(B) / 2$ holds no matter how rapidly $f$ grows, and Cusick later showed the case $B=\mathbb{N}$ and $f(n)=2^{2^{2^{n}}}$ as a counterexample (see [3, Lemma 3]). Nowadays it is known that the Hausdorff dimension of the set

$$
\left\{x \in \mathbb{I}: a_{n}(x) \geq f(n) \text { for all } n \in \mathbb{N}\right\}
$$

decreases as $f$ grows more rapidly. Good proved that it is $1 / 2$ if $f(n)<(\log n)^{A}$ for some $A>0$, for all sufficiently large $n$ (see [7, Theorem 3]). Hirst improved the upper bound of Good on $f$ to certain double exponential functions (see [8, Theorem 2]). For an arbitrary double exponential function $f(n)=c^{b^{n}}$ where $b, c>1$, Euczak [14, Feng et al. [6] showed that the Hausdorff dimension of the set is $\frac{1}{1+b}$, after a partial result of Moorthy [16]. A related result can be found in [12. Another related result is due to Wang and Wu [19, Theorems 3.1 and 4.2] which gives a complete description of the Hausdorff dimension of sets $\{x \in$ $\mathbb{I}: a_{n}(x) \geq f(n)$ for infinitey many $\left.n \in \mathbb{N}\right\}$. For an arbitrary infinite subset $B$ of $\mathbb{N}$ and an arbitrary double exponential function $f$, Cao, Wang and Wu linked $\tau(B)$ to the Hausdorff dimension of $F(B, f)$ (see [1, Theorem 1.2]). In [5, Lemma 3.2], Fan et al. computed the Hausdorff dimension of sets of the form

$$
\left\{x \in \mathbb{I}: s_{n} \leq a_{n}(x)<N s_{n} \text { for all } n \in \mathbb{N}\right\},
$$

where $s_{n} \geq 3, s_{n} \rightarrow \infty$ as $n \rightarrow \infty$ and $N \geq 2$.

In view of these developments, it is also relevant to describe the Hausdorff dimension of subsets of $E(B)$ with slowly growing partial quotients, namely, sets of the form

$$
G(B, f)=\left\{x \in E(B): a_{n}(x) \leq f(n) \text { for all } n \in \mathbb{N}\right\},
$$

where $f$ is a function taking values in $[\min B, \infty)$ and tending to infinity with $n$. Our main result states that the Hausdorff dimension of this set never drops from $\tau(B) / 2$ no matter how slowly $f$ grows. 
Theorem 1. For any infinite subset $B$ of $\mathbb{N}$ and any function $f: \mathbb{N} \rightarrow[\min B, \infty)$ such that $\lim _{n \rightarrow \infty} f(n)=\infty$, the set

$G(B, f)=\left\{x \in \mathbb{I}: a_{n}(x) \in B, a_{n}(x) \leq f(n)\right.$ for all $n \in \mathbb{N}$, and $\left.\lim _{n \rightarrow \infty} a_{n}(x)=\infty\right\}$ is of Hausdorff dimension $\tau(B) / 2$.

Since $E(B)$ contains $G(B, f)$, Theorem 1 yields $\operatorname{dim}_{H} E(B) \geq \tau(B) / 2$. From this and the upper bound $\operatorname{dim}_{H} E(B) \leq \tau(B) / 2$ obtained by Hirst [9, Corollary 1], we obtain $\operatorname{dim}_{H} E(B)=\tau(B) / 2$, a positive answer to the first conjecture of Hirst as proved by Wang and $\mathrm{Wu}$ in [20, Theorem 1.1]. The original proof of Wang and Wu does not use ergodic theory.

By virtue of the upper bound obtained by Hirst [9, Corollary 1], for a proof of Theorem 1 it suffices to show the lower bound $\operatorname{dim}_{H} G(B, f) \geq \tau(B) / 2$. Our proof of this relies on the ergodic theory for the iteration of the Gauss map which generates the partial quotients in the continued fraction expansion. After introducing notations in Section 2, we construct in Section 3 a sequence of Bernoulli measures with non-uniform weights, supported on finitely many 1-cylinders indexed by elements of $B$ and having dimensions (the Kolmogorov-Sinai entropy divided by the Lyapunov exponent, see [15, Theorem 4.4.2]) not much smaller than $\tau(B) / 2$. This construction relies on a novel use of the exponent of convergence, as in (3.1) below. Using these measures, in Section 4 we construct fractal subsets of $G(B, f)$ and Borel probability measures supported on them, and appeal to the mass distribution principle [4] to estimate from below the Hausdorff dimension of these fractal sets.

\section{Notations}

Following the monograph of Khinchin [13] we introduce basic notations. For each $n \in \mathbb{N}$ and $\left(a_{1}, a_{2}, \ldots, a_{n}\right) \in \mathbb{N}^{n}$, we call the interval

$$
I\left(a_{1}, a_{2}, \ldots, a_{n}\right)= \begin{cases}{\left[\frac{p_{n}}{q_{n}}, \frac{p_{n-1}+p_{n}}{q_{n-1}+q_{n}}\right)} & \text { if } n \text { is even, } \\ \left(\frac{p_{n-1}+p_{n}}{q_{n-1}+q_{n}}, \frac{p_{n}}{q_{n}}\right] & \text { if } n \text { is odd }\end{cases}
$$

an $n$-cylinder. Here, $p_{k}, q_{k}(k=0, \ldots, n)$ are given by the recursion formulas

$$
\begin{aligned}
& p_{-1}=1, p_{0}=0, p_{k}=a_{k} p_{k-1}+p_{k-2} \quad \text { for } k=1, \ldots, n, \\
& q_{-1}=0, q_{0}=1, q_{k}=a_{k} q_{k-1}+q_{k-2} \quad \text { for } k=1, \ldots, n .
\end{aligned}
$$

The $n$-cylinder $I\left(a_{1}, a_{2}, \ldots, a_{n}\right)$ represents the set of all real numbers in $[0,1)$ which have a (finite or infinite) continued fraction expansion beginning by $a_{1}, a_{2}, \ldots, a_{n}$, i.e.,

$$
I\left(a_{1}, a_{2}, \ldots, a_{n}\right)=\left\{x \in[0,1): a_{1}(x)=a_{1}, a_{2}(x)=a_{2}, \ldots, a_{n}(x)=a_{n}\right\} .
$$

In the case of 1 -cylinders, note that $I(k)=\left(\frac{1}{k+1}, \frac{1}{k}\right]$ for each $k \in \mathbb{N}$.

We denote $\left(a_{1}, \ldots, a_{n}\right) \in \mathbb{N}^{n}$ simply by $\omega$ and write $I(\omega)$ for the $n$-cylinder $I\left(a_{1}, \ldots, a_{n}\right)$. For $\omega_{1}=\left(a_{1}, \ldots, a_{n}\right) \in \mathbb{N}^{n}$ and $\omega_{2}=\left(b_{1}, \ldots, b_{m}\right) \in \mathbb{N}^{m}$, we write $\left(\omega_{1}, \omega_{2}\right)$ for $\left(a_{1}, \ldots, a_{n}, b_{1}, \ldots, b_{m}\right) \in \mathbb{N}^{n+m}$ and so on. 


\section{MeAsures Supported ON RESTRICTED 1-CYLINDERS}

The continued fraction expansion is generated by iterations of the Gauss map $T:(0,1] \rightarrow[0,1)$ given by

$$
T(x)=\frac{1}{x}-\left\lfloor\frac{1}{x}\right\rfloor
$$

where $\lfloor y\rfloor$ denotes the largest integer not exceeding $y$. For each $x \in \mathbb{I}$ and $n \geq 1$, $a_{n}(x)=\left\lfloor 1 / T^{n-1}(x)\right\rfloor$ holds. We say a Borel probability measure $\mu$ on $[0,1]$ is $T$-invariant if $\mu(\mathbb{I})=1$ and $\mu\left(T^{-1}(A)\right)=A$ holds for any Borel subset $A$ of $\mathbb{I}$. We say a $T$-invariant Borel probability measure $\mu$ is ergodic if $T^{-1}(A)=A$ for a Borel subset $A$ of $\mathbb{I}$ implies $\mu(A)=0$ or $=1$. For a $T$-invariant Borel probability measure $\mu$, let $h(\mu)$ denote the Kolmogorov-Sinai entropy of $\mu$ relative to the restriction of $T$ to $\mathbb{I}$. Since the set of 1-cylinders generates the Borel sigma-algebra of $\mathbb{I}$, we have

$$
h(\mu)=-\lim _{n \rightarrow \infty} \frac{1}{n} \sum_{\left(a_{1}, \ldots, a_{n}\right) \in \mathbb{N}^{n}} \mu\left(I\left(a_{1}, \ldots, a_{n}\right)\right) \log \mu\left(I\left(a_{1}, \ldots, a_{n}\right)\right) \in[0, \infty],
$$

where $0 \log 0:=0$. Define the Lyapunov exponent of $\mu$ relative to $T$ by

$$
\chi(\mu)=\int \log \left|T^{\prime}\right| d \mu \in\left[2 \log \frac{\sqrt{5}-1}{2}, \infty\right] .
$$

Lemma 2. Let $B$ be an infinite subset of $\mathbb{N}$ with $\tau(B)>0$. For any $\epsilon \in(0, \tau(B) / 2)$ there exist a strictly increasing sequence $\left\{b_{m}\right\}_{m \in \mathbb{N}}$ in $B$ and a sequence $\left\{\mu_{m}\right\}_{m \in \mathbb{N}}$ of T-invariant ergodic Borel probability measures on $[0,1]$ with the following properties:

(a) for all $m \in \mathbb{N}$,

$$
\sum_{\substack{k \in B \\ b_{m} \leq k<b_{m+1}}} \mu_{m}(I(k))=1
$$

(b) for all $m \in \mathbb{N}$, both $h\left(\mu_{m}\right)$ and $\chi\left(\mu_{m}\right)$ are finite and

$$
\liminf _{m \rightarrow \infty} \frac{h\left(\mu_{m}\right)}{\chi\left(\mu_{m}\right)} \geq \frac{\tau(B)}{2}-\epsilon .
$$

Proof. Let $\epsilon \in(0, \tau(B) / 2)$. We define a strictly increasing sequence $\left\{b_{m}\right\}_{m \in \mathbb{N}}$ in $B$ inductively as follows: set $b_{1}=\min B$, and $b_{m+1}>b_{m}$ is the minimal integer in $B$ such that

$$
\sum_{\substack{b_{m} \leq k<b_{m+1} \\ k \in B}} k^{-\tau(B)+\epsilon} \geq 1
$$

This definition makes sense since $\tau(B)$ is the exponent of convergence of $B$. Put $B_{m}=\left\{k \in B: b_{m} \leq k<b_{m+1}\right\}$. Let $\mu_{m}$ denote the Bernoulli measure which assigns to each 1-cylinder $I(k), k \in B_{m}$ the probability $Z_{m} k^{-\tau(B)+\epsilon}$, with the normalizing constant $Z_{m}=\left(\sum_{k \in B_{m}} k^{-\tau(B)+\epsilon}\right)^{-1}$. Item (a) is obvious from the definition of $\mu_{m}$. 
Since $\mu_{m}$ is a Bernoulli measure, it is $T$-invariant and ergodic. The entropy of $\mu_{m}$ is given by

$$
h\left(\mu_{m}\right)=-\sum_{k \in B_{m}} \mu(I(k)) \log \mu(I(k))
$$

Then

$$
\begin{aligned}
h\left(\mu_{m}\right) & =-\sum_{k \in B_{m}} Z_{m} k^{-\tau(B)+\epsilon} \log \left(Z_{m} k^{-\tau(B)+\epsilon}\right) \\
& =-\log Z_{m}+(\tau(B)-\epsilon) Z_{m} \sum_{k \in B_{m}} k^{-\tau(B)+\epsilon} \log k \\
& \geq(\tau(B)-\epsilon) Z_{m} \sum_{k \in B_{m}} k^{-\tau(B)+\epsilon} \log k,
\end{aligned}
$$

because of $Z_{m} \leq 1$ from (3.1). For the Lyapunov exponent of $\mu_{m}$ we have

$$
\chi\left(\mu_{m}\right)=2 \int|\log x| d \mu_{m}(x) \leq 2 Z_{m} \sum_{k \in B_{m}} k^{-\tau(B)+\epsilon} \log (k+1) .
$$

From (3.2) and (3.3), for all sufficiently large $m$ we have

$$
\frac{h\left(\mu_{m}\right)}{\chi\left(\mu_{m}\right)} \geq \frac{1}{2}(\tau(B)-\epsilon) \min _{k \in B_{m}} \frac{\log k}{\log (k+1)} \geq \frac{1}{2} \tau(B)-\epsilon,
$$

which implies (b).

\section{Proof of Theorem 1 .}

Let $B$ be an infinite subset of $\mathbb{N}$ and $f: \mathbb{N} \rightarrow[\min B, \infty)$ be a function such that $\lim _{n \rightarrow \infty} f(n)=\infty$. By virtue of the upper bound obtained by Hirst [9, Corollary 1], it is enough to show the lower bound

$$
\operatorname{dim}_{H} G(B, f) \geq \frac{1}{2} \tau(B) .
$$

We assume $\tau(B)>0$, for otherwise there is nothing to prove. For any number strictly less than $\tau(B) / 2$, we will construct a subset of $G(B, f)$ whose Hausdorff dimension exceeds that number.

Let $\epsilon \in(0, \tau(B) / 2)$. Let $\left\{b_{m}\right\}_{m \in \mathbb{N}}$ be a strictly monotone increasing sequence in $B$ and let $\left\{\mu_{m}\right\}_{m \in \mathbb{N}}$ be a sequence of $T$-invariant ergodic Borel probability measures for which the conclusions of Lemma 2 hold. Set

$$
B_{m}= \begin{cases}\{\min B\} & \text { for } m=1 . \\ \left\{k \in B: b_{m} \leq k<b_{m+1}\right\} & \text { for } m \geq 2 .\end{cases}
$$

For each $m \geq 1$ and an integer $\ell \geq 1$ we define

$$
B_{m}^{\ell}=\left\{\left(a_{1}, \ldots, a_{\ell}\right) \in \mathbb{N}^{\ell}: a_{i} \in B_{m} \quad \text { for } i=1, \ldots, \ell\right\} .
$$

Put $\ell_{1}=1$ and $A_{1}^{\ell_{1}}=B_{1}$ for ease of notation. Let $m \geq 2$. From Birkhoff's ergodic theorem and Shannon-McMillan-Breiman's theorem (see e.g. [2]) applied to the measure $\mu_{m}$, and the fact (see [2, Chapter $\left.7, \S 4\right]$ ) that there exists a constant $C>1$ such that $\left|\left(T^{n}\right)^{\prime}(x)\right| /\left|\left(T^{n}\right)^{\prime}(y)\right| \leq C$ holds for every $n \geq 1$ and all $x, y \in(0,1]$ 
contained in the same $n$-cylinder, there exist an integer $\ell_{m} \geq 1$ and a subset $A_{m}^{\ell_{m}}$ of $B_{m}^{\ell_{m}}$ such that

$$
\left|\frac{1}{\ell_{m}} \log \# A_{m}^{\ell_{m}}-h\left(\mu_{m}\right)\right| \leq \frac{1}{m}
$$

and

$$
\left|\frac{1}{\ell_{m}} \log \right|\left(T^{\ell_{m}}\right)^{\prime}(x)\left|-\chi\left(\mu_{m}\right)\right| \leq \frac{1}{m} \quad \text { for any } \quad x \in \bigcup_{\omega \in A_{m}^{\ell_{m}}} I(\omega),
$$

where the symbol \# denotes the cardinality of sets. Following the notation in the end of Section 2 , for each $m \geq 1$ and $t \geq 1$ put

$$
A_{m}^{t \ell_{m}}=\left\{\left(\omega_{1}, \ldots, \omega_{t}\right) \in \mathbb{N}^{t \ell_{m}}: \omega_{i} \in A_{m}^{\ell_{m}} \quad \text { for } i=1, \ldots, t\right\} .
$$

Let $\left\{t_{m}\right\}_{m \in \mathbb{N}}$ be a sequence of positive integers such that for every $m \geq 2$ we have

$$
b_{m+1} \leq \inf \left\{f(n): \sum_{j=1}^{m-1} t_{j} \ell_{j}+1 \leq n \leq \sum_{j=1}^{m} t_{j} \ell_{j}\right\} .
$$

Since $\lim _{n \rightarrow \infty} f(n)=\infty$, one can choose such a sequence by induction on $m$.

We represent each integer $M \geq t_{1}$ as

$$
M=t_{1}+\cdots+t_{m}+s, 0 \leq s \leq t_{m+1}-1,
$$

and introduce a finite subset of $\mathbb{N}^{t_{1} \ell_{1}+\cdots+t_{m} \ell_{m}+s \ell_{m+1}}$ as

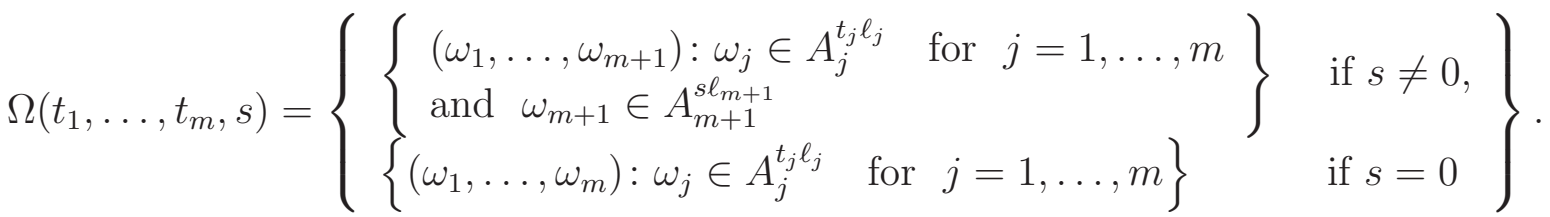

Set

$$
\Lambda=\bigcap_{M=t_{1}}^{\infty} \bigcup_{\omega \in \Omega\left(t_{1}, \ldots, t_{m}, s\right)} I(\omega) .
$$

The set $\Lambda$ is an intersection of decreasing compact sets, and so a non-empty compact set. By construction we have

$$
E(B) \supset \Lambda \cap \mathbb{I} \text {. }
$$

Let $x \in \Lambda \cap \mathbb{I}$. By construction, for each $m \geq 1$ and $0 \leq s \leq t_{m+1}-1$ there exists $\omega \in \Omega\left(t_{1}, \ldots, t_{m}, s\right)$ such that $x \in I(\omega)$. The first alternative of (4.2) gives

$$
a_{n}(x)=\min B \leq f(n) \text { for } n=1, \ldots, t_{1} \ell_{1} .
$$

For every $m \geq 2$, the second alternative of (4.2) and (4.5) yield

$$
a_{n}(x)<b_{m+1} \leq f(n) \text { for } n=\sum_{j=1}^{m-1} t_{j} \ell_{j}+1, \ldots, \sum_{j=1}^{m} t_{j} \ell_{j} .
$$

It follows that $x \in G(B, f)$, and therefore

$$
G(B, f) \supset \Lambda \cap \mathbb{I} .
$$


For each $\omega \in \Omega\left(t_{1}, \ldots, t_{m}, s\right)$ fix a point $x(\omega) \in I(\omega) \cap \Lambda$. Let $\nu_{M}$ denote the uniform probability distribution on the finite set $\bigcup\left\{x(\omega): \omega \in \Omega\left(t_{1}, \ldots, t_{m}, s\right)\right\}$, namely $\nu_{M}=\left(1 / \# \Omega\left(t_{1}, \ldots, t_{m}, s\right)\right) \sum_{\omega \in \Omega\left(t_{1}, \ldots, t_{m}, s\right)} \delta_{x(\omega)}$ where $\delta_{x(\omega)}$ denotes the unit point mass at $x(\omega)$. Let $\nu$ be an accumulation point of the sequence $\left\{\nu_{M}\right\}_{M \geq t_{1}}$ in the weak* topology on the space of Borel probability measures on $[0,1]$. We claim that $\nu(\Lambda)=1$. Indeed, since $\nu$ is regular (see [18, Theorem 6.1]) and $\Lambda$ is compact, $\nu(\Lambda)<1$ would imply the existence of an open set $C \subset[0,1]$ such that $C \cap \Lambda=\emptyset$ and $\nu(C)>0$. Since $\nu_{M}(C) \leq \nu_{M}([0,1] \backslash \Lambda)=0$ for all $M$, it would follow that $\nu(C) \leq \liminf _{M \rightarrow \infty} \nu_{M}(C)=0$, a contradiction. We show that

$$
\liminf _{r \rightarrow 0} \frac{\log \nu(B(x, r) \cap \Lambda)}{\log r} \geq \liminf _{m \rightarrow \infty} \frac{h\left(\mu_{m}\right)}{\chi\left(\mu_{m}\right)} \text { for all } x \in \Lambda,
$$

where $B(x, r)=(x-r, x+r)$. Then, from the mass distribution principle [4, p.60] and Lemma 2(b) it follows that

$$
\operatorname{dim}_{H} \Lambda \geq \liminf _{m \rightarrow \infty} \frac{h\left(\mu_{m}\right)}{\chi\left(\mu_{m}\right)} \geq \frac{1}{2} \tau(B)-\epsilon .
$$

Since $\epsilon \in(0, \tau(B) / 2)$ is arbitrary, we obtain (4.1).

The rest of this paper is devoted to the proof of (4.7). For each integer $M \geq t_{1}$ in (4.6), we set

$$
r_{M}=e^{-t_{m} \ell_{m}\left(\chi\left(\mu_{m}\right)+\frac{3}{m}\right)-s \ell_{m+1}\left(\chi\left(\mu_{m+1}\right)+\frac{2}{m+1}\right)} .
$$

We choose $t_{m}(m=1,2, \ldots)$ inductively so that $r_{M}$ is strictly monotone decreasing and converges to 0 as $M \rightarrow \infty$.

Let $r \in\left(0, t_{1}\right]$, and let $M \geq t_{1}$ be such that

$$
r_{M+1}<r \leq r_{M}
$$

For each $x \in \Lambda$ we estimate $\nu(B(x, r) \cap \Lambda)$ from above. Let $|J|$ denote the Euclidean length of a bounded interval $J$. For each $\omega \in \Omega\left(t_{1}, \ldots, t_{m}, s\right)$ we have

$$
\begin{aligned}
\sup _{I(\omega)} \log \left|\left(T^{\sum_{j=1}^{m} t_{j} \ell_{j}+s \ell_{m+1}}\right)^{\prime}\right| & \leq \sum_{j=1}^{m} t_{j} \ell_{j}\left(\chi\left(\mu_{j}\right)+\frac{1}{j}\right)+s \ell_{m+1}\left(\chi\left(\mu_{m+1}\right)+\frac{1}{m+1}\right) \\
& \leq t_{m} \ell_{m}\left(\chi\left(\mu_{m}\right)+\frac{2}{m}\right)+s \ell_{m+1}\left(\chi\left(\mu_{m+1}\right)+\frac{1}{m+1}\right) \\
& \leq \log \frac{1}{r_{M}} .
\end{aligned}
$$

The first inequality follows from (4.4). The second one holds provided $t_{m}$ is chosen to be large enough compared to $t_{1}, \ldots, t_{m-1}$. The last one is by (4.8). Using the mean value theorem and the above estimate of the derivative,

$$
|I(\omega)| \geq \frac{\left|T^{\sum_{j=1}^{m} t_{j} \ell_{j}+s \ell_{m+1}}(I(\omega))\right|}{\sup _{I(\omega)}\left|\left(T^{\sum_{j=1}^{m} t_{j} \ell_{j}+s \ell_{m+1}}\right)^{\prime}\right|}=\frac{1}{\sup _{I(\omega)}\left|\left(T^{\sum_{j=1}^{m} t_{j} \ell_{j}+s \ell_{m+1}}\right)^{\prime}\right|} \geq r_{M} .
$$

Since $r \leq r_{M}$, this lower bound immediately gives an upper bound on the number of intervals $I(\omega)$ which intersect a fixed interval of length $2 r$ intersecting $\Lambda$. For 
any $x \in \Lambda$ we have

$$
\begin{aligned}
\#\left\{\omega \in \Omega\left(t_{1}, \ldots, t_{m}, s\right): B(x, r) \cap I(\omega) \neq \emptyset\right\} & \leq \frac{2 r_{M}}{\inf _{\omega \in \Omega\left(t_{1}, \ldots, t_{m}, s\right)}|I(\omega)|}+2 \\
& \leq 4 .
\end{aligned}
$$

Let $M^{\prime}=t_{1}+\cdots+t_{m^{\prime}}+s^{\prime}$ be an integer with $m^{\prime} \geq m, 0 \leq s^{\prime} \leq t_{m^{\prime}+1}-1$ and $M^{\prime} \geq$ $M+2$. If $\omega \in \Omega\left(t_{1}, \ldots, t_{m}, s\right)$ and $\omega^{\prime} \in \Omega\left(t_{1}, \ldots, t_{m^{\prime}}, s^{\prime}\right)$, then $\overline{I(\omega)} \cap \overline{I\left(\omega^{\prime}\right)}=\emptyset$, or $\overline{I\left(\omega^{\prime}\right)}$ is contained in the interior of $I(\omega)$. In particular, for any $\omega \in \Omega\left(t_{1}, \ldots, t_{m}, s\right)$, $\partial I(\omega) \cap \Lambda=\emptyset$ holds. Since $\nu(\Lambda)=1$ we have $\nu(\partial I(\omega))=0$. Moreover, for every $q \geq M$, the construction gives

$$
\nu_{q}(I(\omega))=\frac{1}{\left(\# A_{1}^{\ell_{1}}\right)^{t_{1}} \cdots\left(\# A_{m}^{\ell_{m}}\right)^{t_{m}}\left(\# A_{m+1}^{\ell_{m+1}}\right)^{s}} \leq \frac{1}{\left(\# A_{m}^{\ell_{m}}\right)^{t_{m}}\left(\# A_{m+1}^{\ell_{m}}\right)^{s}} .
$$

The weak* convergence of Borel probability measures on $[0,1]$ gives

$$
\nu(I(\omega))=\lim _{q \rightarrow \infty} \nu_{q}(I(\omega)) \leq \frac{1}{\left(\# A_{m}^{\ell_{m}}\right)^{t_{m}}\left(\# A_{m+1}^{\ell_{m+1}}\right)^{s}} .
$$

By (4.3) and (4.10), for any $x \in \Lambda$ we have

$$
\nu(B(x, r) \cap \Lambda) \leq 4 e^{-t_{m} \ell_{m}\left(h\left(\mu_{m}\right)-\frac{1}{m}\right)} e^{-s \ell_{m+1}\left(h\left(\mu_{m+1}\right)-\frac{1}{m+1}\right)} .
$$

If $0 \leq s<t_{m+1}-1$ then $M+1=t_{1}+\cdots+t_{m}+s+1$ by (4.6), and $r_{M+1}=$ $e^{-\ell_{m+1}\left(\chi\left(\mu_{m+1}\right)+\frac{2}{m+1}\right)} r_{M}$ by (4.8). From this and (4.11),

$$
\begin{aligned}
\frac{\log \nu(B(x, r) \cap \Lambda)}{\log r_{M+1}} \geq & \frac{t_{m} \ell_{m}\left(h\left(\mu_{m}\right)-1 / m\right)+s \ell_{m+1}\left(h\left(\mu_{m+1}\right)-1 /(m+1)\right)}{t_{m} \ell_{m}\left(\chi\left(\mu_{m}\right)+3 / m\right)+(s+1) \ell_{m+1}\left(\chi\left(\mu_{m+1}\right)+2 /(m+1)\right)} \\
& +O\left(\frac{1}{|\log r|}\right) .
\end{aligned}
$$

Since $\liminf _{m \rightarrow \infty} h\left(\mu_{m}\right)>0$, the two terms in the numerator of the fraction are positive for any sufficiently large $m$. If necessary, we replace $t_{m}$ by a sufficiently large integer so that for $0 \leq s<m$, the first term in the denominator of the fraction dominates the second one so that

$$
\frac{\log \nu(B(x, r) \cap \Lambda)}{\log r_{M+1}} \geq \frac{h\left(\mu_{m}\right)-1 / m}{\chi\left(\mu_{m}\right)+4 / m}+O\left(\frac{1}{|\log r|}\right) .
$$

If $m \leq s<t_{m+1}-1$, then we clearly have

$$
\frac{\log \nu(B(x, r) \cap \Lambda)}{\log r_{M+1}} \geq \min \left\{\frac{h\left(\mu_{m}\right)-1 / m}{\chi\left(\mu_{m}\right)+3 / m}, \frac{m\left(h\left(\mu_{m+1}\right)-1 /(m+1)\right)}{(m+1)\left(\chi\left(\mu_{m+1}\right)+2 /(m+1)\right)}\right\}+O\left(\frac{1}{|\log r|}\right) .
$$

In the remaining case $s=t_{m+1}-1$, we have $M+1=t_{1}+\cdots+t_{m+1}$ by (4.6) and $r_{M+1}=e^{-t_{m+1} \ell_{m+1}\left(\chi\left(\mu_{m+1}\right)+\frac{3}{m+1}\right)}$ by (4.8). Using this and (4.11),

$$
\frac{\log \nu(B(x, r) \cap \Lambda)}{\log r_{M+1}} \geq \frac{\left(t_{m+1}-1\right)\left(h\left(\mu_{m+1}\right)-1 /(m+1)\right)}{t_{m+1}\left(\chi\left(\mu_{m+1}\right)+2 /(m+1)\right)}+O\left(\frac{1}{|\log r|}\right) .
$$

As $r \rightarrow 0$ we have $M \rightarrow \infty$ and $r_{M} \rightarrow 0$ by (4.9), and (4.7) follows. This completes the proof of Theorem 1 . 
Acknowledgments. I thank anonymous referees for their careful readings of the manuscript and giving useful suggestions for improvements. I thank Johannes Jaerisch for fruitful discussions. This research was partially supported by the JSPS KAKENHI 19K21835, $20 \mathrm{H} 01811$.

\section{REFERENCES}

[1] Chun-Yun Cao, Bao-Wei Wang and Jun Wu, The growth speed of digits in infinite iterated function systems. Studia Math. 217 (2013) 139-158.

[2] I. P. Cornfeld, S. V. Fomin and Ya. G. Sinai, Ergodic Theory. Grundlehren der mathematischen Wissenschaften 245 Springer 1982

[3] Thomas W. Cusick, Hausdorff dimension of sets of continued fractions. Quart. J. Math. Oxford, 41 (1990) 277-286.

[4] Kenneth Falconer, Fractal Geometry (Mathematical foundations and applications). Second edition. Wiley, Chichester, 2003

[5] Ai-Hua Fan, Ling-Min Liao, Bao-Wei Wang and Jun Wu, On Khintchine exponents and Lyapunov exponents of continued fractions. Ergodic Theory Dynam. Systems 29 (2009) 73-109.

[6] De-Jun Feng, Jun Wu, Jyh-Ching Liang and Shiojenn Tseng, Appendix to the paper by T. Euczak- A simple proof of the lower bound: "On the fractional dimension of sets of continued fractions". Mathematika 44 (1997) 54-55.

[7] Irving John Good, The fractional dimensional theory of continued fractions. Proc. Cambridge Philos. Soc. 37 (1941) 199-228.

[8] Keith E. Hirst, A problem in the fractional dimension theory of continued fractions. Quart. J. Math. Oxford, 21 (1970) 29-35.

[9] Keith E. Hirst, Continued fractions with sequences of partial quotients. Proc. Amer. Math. Soc. 38 (1973) 221-227.

[10] Johannes Jaerisch and Marc Kesseböhmer, The arithmetic-geometric scaling spectrum for continued fractions. Ark. Mat. 48 (2010) 335-360.

[11] Vojtěck Jarník, Zur metrischen theorie der diophantischen approximationen. Práce Mat. Fiz. 36 (1928) 91-106.

[12] Thomas Jordan and Michał Rams, Increasing digit subsystems of infinite iterated function systems. Proc. Amer. Math. Soc. 140 (2012) 1267-1279.

[13] Aleksandr Yakovlevich Khinchin, Continued fractions. University of Chicago Press, III.London, 1964.

[14] Tomasz Łuczak, On the fractional dimension of sets of continued fractions. Mathematika 44 (1997) 50-53.

[15] R. Daniel Mauldin and Mariusz Urbański, Graph directed Markov systems: Geometry and Dynamics of Limit Sets. Cambridge Tracts in Mathematics 148 Cambridge University Press (2003)

[16] Chinnadurai Ganesa Moorthy, A problem of Good on Hausdorff dimension. Mathematika 39 (1992) 244-246.

[17] Gerhard Ramharter, Eine Bemerkung über gewisse Nullmengen von Kettenbrüchen. Ann. Univ. Sci. Budapest. Eötvös Sect. Math. 28 (1985) 11-15.

[18] Peter Walters, An Introduction to Ergodic Theory. Graduate Texts in Mathematics 79, Springer-Verlag, New York, 1982.

[19] Bao-Wei Wang and Jun Wu, Hausdorff dimension of certain sets arising in continued fraction expansions. Adv. Math. 218 (2008) 1319-1339.

[20] Bao-Wei Wang and Jun Wu, A problem of Hirst on continued fractions with sequences of partial quotients. Bull. London Math. Soc. 40 (2008) 18-22. 
Keio Institute of Pure and Applied Sciences (KiPAS), Department of Mathematics, Keio University, YoKohama, 223-8522, JAPAN

Email address: hiroki@math.keio.ac.jp

$U R L:$ http://www.math.keio.ac.jp/ ${ }^{\sim h}$ iroki/ 